\title{
Wandlung von Sprache und Tun im Lauf eines ärztlichen Lebens, Inserate sie spiegeln und auch der Kollegen Artikel [1, 2]
}

Gerne würde Walter Welle wechseln seine Arbeitsstelle. Mit schon hoher Facharztreife steht er in der Warteschleife voller Leistungsdrang bereit für die Kadertätigkeit. Auch sein Chef ihm attestiert, fachlich sei er sehr versiert, Er verkehre schrift- und mündlich mit dem Hausarzt freund- und gründlich, sorgsam halt er Mass bei Kosten, wäge ab bei jedem Posten, wende nicht nur hie und da an auch die Generika, sondern wandle jederzeit auf dem Pfad der Sparsamkeit. Deshalb sei man wohl beraten mit so einem Kandidaten. Der, noch spät des Abends hat aufgemacht das Ärzteblatt bei den Inserateseiten mit den Stellenneuigkeiten. Aber diese wurden Welle zu des ernsten Zweifels Quelle an sich selber und, oh weh, auch am eignen Métier.

Denn er las:

«In den Dienst an unsern Kunden fühlen Sie sich eingebunden, weil wir die Klienten schätzen und wozu wir Standards setzen. Human Faktor-Kompetenz steigert Ihre Effizienz, deshalb sehn Sie überall noch verborgenes Potenzial mit der Absicht, zu ergänzen unsres Zentrums Marktvalenzen also denken fürs Spital stets Sie mehrdimensional, dementsprechend breit vernetzt wird das Neue umgesetzt, mit dem Schub der Energie aus der Freizeitsynergie (Unser Haus ist sehr begehrt auch dank seinem Freizeitwert!); orientiert am Budgetziel, runden ab Sie Ihr Profil.»

«Dank dem fachlichen Geschick wahren Sie den Überblick, wirken mit viel Fantasie nicht nur in der Strategie, sondern auch und permanent fest am Zukunftsmanagement.
Und Sie sind ein transparenter

Player in dem Profit-Center; ähnlich seh'n Konzepte aus für den Trakt Gesundheitshaus».

Auch: «Sie haben Visionen für die Innovationen, die die Effizienz ergeben, um dynamisch aufzustreben, denn die Kunden, die wir wollen, schöpfen schliesslich aus dem Vollen!»

Endlich: «Dank Belastbarkeit Ihrer Arztpersönlichkeit können jung Sie, doch erfahren Qualität mit Sparen paaren, denn hier winken sehr probate lohnende Zertifikate nach den Regeln des Vereins ISO-Norm 9001.»

«Interessiert? - wenn ja, sodann sind genau Sie unser Mann! Näher gibt zu diesem Zweck Auskunft Dr. iur. et oec. ... bürozeitlich hilft auch weiter unser * HR-Mitarbeiter.»

Mundtot fand Kollege Welle sich an der Verzweiflung Schwelle, konnte nach so starken Worten nur noch Schwächen bei sich orten, fort war seines Lebenstraumes heiles Bild des Wirkungsraumes, wo er Linderung könnt' bringen und wo Heilung möcht' gelingen einem Arzt mit Nächstenliebe auch in dem Spitalbetriebe Just im selben Augenblick wacht' er auf zu seinem Glück, schweissgebadet, doch er nahm dankbar wahr, dass er entkam einer Unbill, die zuletzt ihn in Angst und Not versetzt', sprach, nach dem Erlebten sinnend und nach seiner Fassung ringend: «Nie hab solches ich gelesen, ist ein Albtraum nur gewesen.»

Sprach's erinnert an Bewährtes seit Jahrhunderten Gelehrtes wie im mahnenden Gedanken Bleulers: «Bleiben wir am Kranken!» [1]
Prof. em. Dr. med.

Max Stäubli

Lebernhöhe 4

CH-8123 Ebmatingen

maxstaeubli[at]bluewin.ch 